\title{
Problematizing "cold" with K12 science teachers
}

\author{
Carolina Alvarado ${ }^{1}$, Michael C. Wittmann ${ }^{1,2}$, Adam Z. Rogers ${ }^{1}$, and Laura Millay ${ }^{1}$ \\ ${ }^{1}$ Center of Research in STEM Education, University of Maine, 5727 Estabrooke Hall, Orono, ME 04469-5709 \\ ${ }^{2}$ Department of Physics, University of Maine, 5709 Bennett Hall, Orono, ME 04469-5709
}

\begin{abstract}
In the Maine Physical Sciences Partnership (MainePSP), we have observed that students improve the way they analyze thermal energy after instruction. Still, many of them continue to use the idea that "coldness" transfers. Past researchers have identified that "cold" is commonly perceived as a separate heat energy. Nevertheless, we have not found specific activities to address this idea. We present analysis of students' conceptual understanding of energy transfer and how the use of coldness as an entity plays a role in it. We explore how both ideas interact with each other using two different multiple choice items. To illustrate the difficulty of addressing student difficulties with coldness, we analyze a collaborative session among K12 teachers who modeled energy transfers in scenarios similar to the student items and had to work to reconcile the conflict between the two models. Our study shows how the concept of coldness as an energy entity can co-exist and be in conflict with the idea of thermal energy, even after instruction.
\end{abstract}

\section{INTRODUCTION}

Energy is an abstract concept that is introduced to children at an early stage and is commonly used in a colloquial manner. There are different ways to address energy using different ontologies; one of the most used in physics books talks about energy in a manner of a substance. [1,2] According to the NGSS, in middle school we expect students to be able to identify different energy forms, transformation of energy, transfer of energy, and conservation of energy [3]. The idea of energy-as-a-substance has been found to address these ideas [4].

One of the scenarios regarding energy to which we are constantly exposed is the exchange of thermal energy or heat. We regularly touch objects that feel warm or cold. A common idea among students is to discuss "coldness" as an energy entity that is transferred in some thermal scenarios [5-8]. This idea of coldness has been observed as a persistent idea that conflicts with the understanding of thermal energy.

In this study we explore how formal instruction of thermal energy in middle school affects the use of the idea of coldness as an energy entity. Our first research question concerns student thinking: (1) Are students consistent in using thermal energy, rather than coldness, after instruction? In order to understand in more detail how energy can be modeled when interacting with cold objects, we explore how teachers model energy for the scenarios used in the students' assessment. Our second research question concerns teacher thinking: (2) How do teachers represent models of thermal energy transfer between warm and cold objects? As we answer this second question, we also attend to how students make sense of coldness and how teachers think about student reasoning,
This study provides insight into how formal instruction can address the idea of coldness as an energy entity that is transferred. By exploring the models of teachers, who are given the task to address these ideas, we analyze more closely the diverse models of thermal interactions and some ideas that might emerge during that process. We provide an insight into the interaction of thermal energy and coldness as an energy entity that can inform future interventions.

\section{RESEARCH SETTING}

The Maine Physical Sciences Partnership (MainePSP) has created a community of in-service science teachers in rural Maine by using a common set of active learning activities to implement in their classrooms. Teachers engage in professional development sessions to improve their understanding of physical sciences content, recognize students' ideas to inform their strategies in the classroom, and participate in evaluation of their students' learning process as well as changes in their own thinking throughout their participation in the process.

The MainePSP has developed assessment instruments to evaluate students' conceptual understanding prior to and post instruction that allow us to observe the effects of instruction. We have used the classroom assessment results from our community in collaborative sessions with teachers to generate discussions regarding persistent incorrect ideas and to explore how to address them. Teachers are now familiar with analyzing students' data and engaging in group conversations that explore the implications of alternative responses in order to understand what students are thinking when they respond in ways that are consistent with incorrect models of energy. 


\section{THERMAL ENERGY VS. COLDNESS}

To assess how students use the idea of thermal energy transfer rather than coldness transfer, we used matched pre-post survey data from two multiple-choice items answered by hundreds of middle school students. Both questions show a scenario where an object interacts with ice or cold ice water. For each question, students get to choose the option they consider most appropriate from two options that use the idea of thermal energy being transferred and two other options that use the idea of coldness being transferred.

The questions were used to start a conversation with a group of teachers to discuss how ideas about coldness or thermal energy transfer are used in their classrooms. After initial remarks about using both ideas were briefly shared, we provided teachers with materials to work in small groups to re-create the two scenarios and model energy transfer in each one. Once they had a model, we shared the students' results for the particular scenario allowing teachers to explore the data. They then worked with the second scenario following the same format.

\section{SURVEY ANALYSIS}

For the analysis, we used matched data, meaning that for the analysis we used only the responses of students who responded both times, giving us a sample of 447 students. We refer to the two items as the Sue Question [9] and the Soda Bucket Question [10] (see
Figure 1). Notably, the two questions used different language to describe similar concepts - "heat" in the Sue Question and "thermal energy" in the Soda Bucket Question. Results show very different use of the idea of thermal energy (heat) and cold. For the analysis, we are interested not only in the number of students who get the question right, but also in contrasting it with the number of students who use the idea of thermal energy or heat being transferred. We use colors to easily identify the two ideas in the response choices as well as in the graphs.

Both before and after instruction, roughly $20 \%$ more students answer the Soda Bucket Question correctly than the Sue Question. The Soda Bucket Question seems less difficult for the students. Furthermore, after instruction, $60 \%$ of the students give an answer involving coldness on the Sue Question, while fewer than 30\% answer the Soda Bucket Question using coldness. In discussions with teachers, described below, we wanted to know how they explain why there might be such great differences in student responses.

Students were also inconsistent in their use of thermal energy (or heat) and coldness in their answers. We analyzed student post-instruction responses to both questions (see Table 1). Only 50\% of the students kept a consistent idea of heat (or thermal energy) being transferred or cold being transferred. The most popular response pattern (given by $40 \%$ of the students) was to answer the Sue Question with the idea that cold is transferred and answer the Soda Can with the idea that thermal energy is transferred.

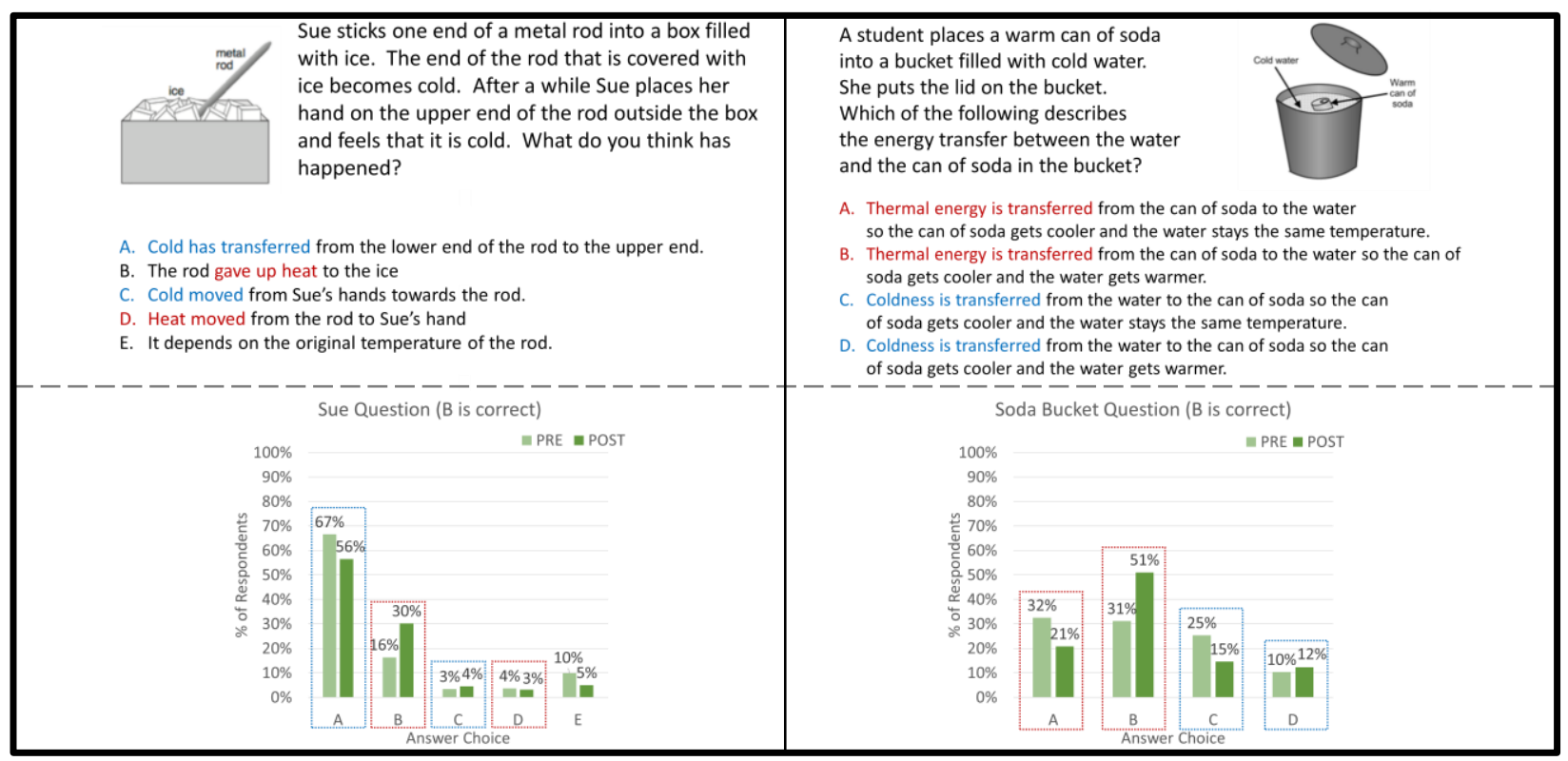

FIG. 1. Two items were used to assess transfer of energy in middle school. We identify in red responses using the idea of thermal energy, and with blue those with coldness being transferred. In the lower part we show the frequency of the responses both pre and post instruction, identifying again whether it uses thermal or cold energy. 
TABLE 1. Post-instruction uses of ideas for both questions.

\begin{tabular}{|l|l|r|r|}
\hline \multirow{2}{*}{ Question } & \multicolumn{2}{c|}{ Soda Can } \\
\cline { 2 - 4 } & Idea used & Heat & Cold \\
\hline \multirow{2}{*}{ Sue } & Heat & $30 \%$ & $5 \%$ \\
\cline { 2 - 4 } & Cold & $40 \%$ & $20 \%$ \\
\hline
\end{tabular}

One could say that the instruction students are receiving is helping them develop the idea of thermal energy - but this problem exists in the pretest data, already. Before instruction, there was a gross disparity in the use of "cold" between the two questions, where $70 \%$ answered with "cold" in the Sue Question, and only 35\% answered with "cold" in the Soda Bucket Question. Instruction is not helping resolve the issue for students, and they are still inconsistent after instruction. Both ideas can co-exist in students' conceptual understanding. We shared these results with our teachers to ask for their perspectives on this issue.

\section{TEACHERS' REPRESENTATIONS OF ENERGY}

Consistent with the culture of the MainePSP, we used the data collected from students to create a space for teachers to discuss the data and their implications. We invited teachers from our community to an evening "collaborative" to discuss the problematic use of coldness as an energy entity. We held two sessions in separate locations. Teachers were given the opportunity to develop their own models of energy being transferred in hands-on scenarios similar to the ones depicted in the student survey. We collected video and audio from the two sessions, as well as the diagrams created by the teachers.

We used these diagrams to analyze how teachers think about thermal energy being transferred, and how this explains how items get colder or hotter. We analyze the statements teachers made while developing their diagrams, with the aim of observing how the ideas of thermal energy and coldness (as an energy entity) are used. At times, teachers brought up events from their classrooms, suggesting that they were aware of the ideas that could be observed in their own classrooms.

\section{A. Teachers' models of energy}

We observed two frameworks about energy flow in the teacher-created representations: thermal energy (heat) moving or coldness moving. Most of the representations focused on thermal energy moving. In Figure 2, we show three different representations of thermal energy moving. On the left, we observe a focus on the directionality of the energy transfer. The arrows show the direction in which thermal energy is transferred from the soda can to the cold water. The picture in the center focuses on the vibration of the molecules (indicated by lines around the molecules).
Objects that are hotter vibrate more while colder objects vibrate less. This particular group kept track of the amount of lines used in the diagram, as an indicator of energy, to illustrate the energy at two different times. The picture on the right uses thermal energy units $(\mathrm{T})$ to illustrate the different amount of energy in the objects. An object that has a larger number of energy units would have a higher temperature. This shows different approaches of addressing thermal interactions focusing on heat being transferred, letting the teachers explain how an object gets colder or hotter.
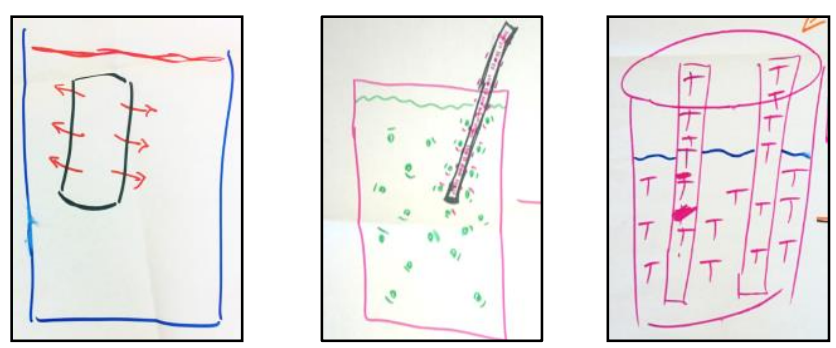

FIG. 2. Teacher models of energy flow from a warm rod to cold water. Diagrams represent flow, energy as the wiggling of molecules, or abstract units of energy, from left to right.

In general, teachers' representations and discussions demonstrated that the teachers in our group understood energy transfer at a level deeper than their students were expected to understand.

\section{B. Hot and cold, heat flow and coldness flow}

While teachers showed strong content knowledge, they used both "heat flow" and "coldness" when discussing these scenarios. In this section, we illustrate two ways that teachers had of talking about energy flow: heat, moving from hotter to colder objects, and coldness, moving from colder to hotter objects. We suggest that some of the difficulties arose because of the lack of clarity with terms describing the temperature (or energy) and the energy flow.

We illustrate the many issues involved by looking at one group's (edited for brevity) conversation. We number the lines to refer to in the subsequent discussion.

1: Hotter temperatures have a greater amount of energy, therefore, the icy-coldness is going to go at the [soda can] 2: Wouldn't the hot move to the- Wouldn't one move to the hot to warm up?

3: I think we need to think about heat loss. Well where is the heat going? Where is the energy going?

4: Well, I think it is going to the snow. And it is going in to melt the snow.

5: So you say that the heat is moving out into the snow, to melt the snow? Do we agree? Do we have a consensus?

6: But the soda is going to get colder too, right? Because the heat is going out. Is that how it works? 
7: Yeah, it is losing heat. That is what is making it colder.

In this discussion, the teachers begin with a description of "icy-coldness" moving into the hotter object (line 1). This suggests both that coldness is a kind of energy and it flows from cold to hot. Some teachers object, raising questions about which process needs to be explained (line 2 and 3). In lines 4 and 5, the discussion is of heat moving from the soda can to the snow. But, a teacher (in line 6, same as in line 1), raises the issue of the soda can getting colder - it seems that the issue is not just how the heat flows (making the snow warmer) but that the coldness of the can needs to be accounted for. The teacher takes the idea of heat going out and accounts for the change in temperature, and in line 7 , this idea is affirmed.

We make three points about this interaction. First, we see teachers thinking about the direction of energy transfer, from the soda can to the snow in the form of thermal energy transfer or heat loss. They considered implications in terms of changes to the temperatures of the objects in the system. The snow would melt, and the can would get colder, both due to the single effect of energy transfer.

Second, and more problematic, we see teachers using terms associated with heat to describe the temperature of the object ("hotter temperatures," line 1), the movement of energy ("heat... moving," line 5), and some combination of the two ("heat loss," line 3, "losing heat," line 7). This result, and the associated confusion between heat and temperature, is well-known in the research literature.

Third, we see evidence of the same lack of clarity about terms associated with cold. In line 1 , the suggestion is that icy-coldness moves into the hotter temperature object, as if it were a kind of energy. (One can imagine not a T, like in the right picture in Figure 2, but a $\mathrm{C}$, for coldness, that travels into the metal rod.) In lines 6 and 7, "colder" is used to describe the temperature of the object. We note the shift between lines 1 and 6, as the teacher first uses "coldness" (as a kind of energy) to account for the soda can getting colder, but then shifts to having "heat going out" to account for the temperature being colder. This reconciles the heat and coldness models in a way that many students have not (as discussed in section IV).

In considering the use of hot, cold, heat, thermal energy, and coldness, we conclude that the language used in the Sue Question and the Soda Bucket Question (Figure 1) is

[1] R. E. Scherr, H. G. Close, S. B. McKagan, and S. Vokos, Phys. Rev. ST Phys. Educ. Res. 8, 020114 (2012).

[2] R. E. Scherr, H. G. Close, E. W. Close, and S. Vokos, Phys. Rev. ST Phys. Educ. Res. 8, 020115 (2012).

[3] Achieve, Inc. (2013).

[4] A. R. Daane, L. Wells, and R. E. Scherr, Phys. Teach. 52, 291 (2014).

[5] H. Georgiou and M. D. Sharma, Int. J. Sci. Math. grossly inconsistent. We note that we have since changed the language of the Sue Question to be consistent with the Soda Bucket Question (for example, not using heat but using thermal energy instead), but that preliminary results show no change in student responses before instruction. The issue of coldness still dominates, suggesting that understanding how objects get colder remains difficult and was not simply an artifact of a badly phrased question.

\section{CONCLUSIONS}

In everyday life we use language that suggests movement of coldness, such as the idea of letting "coldness" come into a house in the winter ("Close the door, you're letting the cold in!"). Students bring these kinds of ideas into the classroom. When it comes to gaining a solid conceptual understanding of transfer, transformation, and conservation of energy in middle school classrooms, considering coldness as a form of energy may be problematic. Our data show that students' thinking about coldness persists after instruction and that many students consider that both thermal energy and coldness are transferred between objects.

This problem has been identified in the literature [11] and was identified by MainePSP teachers as a problem, as well. During teachers' discussions, the challenges presented by everyday language were evident. In teachers' discussions, multiple ideas about thermal energy, heat (as thermal energy), hotness (as temperature), and coldness (as temperature or as an alternative to thermal energy) were discussed and, at times, had to be resolved. There is a lack of current literature regarding instructional strategies to address the topic of "coldness" as a kind of energy. Our study shows that we still need to find effective strategies to address student thinking about coldness.

\section{ACKNOWLEDGMENTS}

This work was supported by the National Science Foundation, MSP-0962805 and DRL-1222580. We thank the MainePSP teacher for their commitment to their students and engagement on looking for a better approach to address coldness in their classroom.

Educ. 10, 1119 (2012).

[6] G. Quan, A. P. Heron, P. Shaffer, and L. Mcdermott, in Phys. REU (Seattle, WA, 2011).

[7] A. S. Rosebery, M. Ogonowski, M. Dischino, and B. Warren, J. Learn. Sci. 19, 322 (2010).

[8] S. Yeo and M. Zadnik, Phys. Teach. 39, 496 (2001).

[9] Mosart Self (2011).

[10] AAAS Science Assessment (2016).

[11] R. Lancor, Sci. Educ. 23, 1245 (2012). 